\title{
Variations in biomarkers of dyslipidemia and dysbiosis during the menstrual cycle: a pilot study in healthy volunteers
}

Helena Bergström ${ }^{\text {**}} \mathbb{D}$, Lena Ekström², Anna Warnqvist ${ }^{3}$, Peter Bergman ${ }^{4,5}$ and Linda Björkhem-Bergman ${ }^{1,6}$

\begin{abstract}
Background: Dyslipidemia in metabolic syndrome may introduce an underestimation of the risk for cardiovascular disease (CVD) using Low-Density Lipoprotein-Cholesterol (LDL-C) as a surrogate marker. Recently, non-High-Density Lipoprotein-Cholesterol (non-HDL-C), Apolipoprotein B (ApoB) and remnant-Cholesterol (remnant-C) have been suggested as better biomarkers for dyslipidemia. In addition, the microbial metabolites trimethylamine- $\mathrm{N}$-oxide (TMAO), betaine and choline have been associated with CVD and suggested as markers for dysbiosis. There is a lack of knowledge on potential alterations in these biomarkers during the menstrual cycle. The aim of this single center, prospective non-interventional study, was to investigate variations in biomarkers of dyslipidemia and dysbiosis in healthy volunteers during the menstrual cycle.
\end{abstract}

Method: Serum samples were collected from 17 healthy, regularly menstruating women during two menstrual cycles, including the follicular, ovulatory and luteal phases. Levels of lipoproteins, lipoprotein ratios and microbial metabolites were analyzed in a total of 90 samples (30 complete menstrual cycles).

Results: $\mathrm{ApoB}, \mathrm{ApoB} / \mathrm{HDL}$ and non-HDL-C/HDL ratios were significantly higher in the follicular phase compared to the ovulatory and luteal phases $(p<0.05)$. Remnant- $C$ were higher during the luteal phase $(p<0.05)$. TMAO did not vary during the different phases and did not correlate with estrogen levels.

Conclusion: Our data support that biomarkers for dyslipidemia vary during the menstrual cycle. Thus, to avoid an underestimation of cardiovascular risk, sampling during the follicular phase, when levels of pro-atherogenic lipids are higher, may be considered.

Keywords: Cardiovascular disease, Atherosclerosis, Menstrual cycle, Dyslipidemia, Apolipoprotein B (ApoB), NonHigh-Density Lipoprotein-Cholesterol (non-HDL-C), Remnant-Cholesterol (remnant-C), Dysbiosis, Gut microbiota, Trimethylamine N-oxide (TMAO)

\section{Background}

During the last decades, there has been a steady rise in the prevalence of the cardiometabolic risk factors known as metabolic syndrome (MetS) [1-3]. Globally, obesity

\footnotetext{
*Correspondence: helena.bergstrom.1@ki.se

${ }^{1}$ Division of Clinical Geriatrics, Department of Neurobiology, Care Sciences and Society (NVS), Karolinska Institute, Blickagången 16, Neo floor 7, 14183 Huddinge, Sweden

Full list of author information is available at the end of the article
}

$\left(\mathrm{BMI}>30 \mathrm{~kg} / \mathrm{m}^{2}\right)$ is now more common in women than men $(15 \%$ vs $11 \%)$. Concomitantly, while the prevalence of acute myocardial infarction (AMI) has decreased in men aged 35-54 years, it has increased in women in midlife $[1,4]$.

Not surprisingly, there are also sex differences in gut microbiota [5] and in the age-adjusted prevalence of MetS. In addition, dysbiosis, an imbalance of the gut 
bacteria, has been associated with the MetS and with estrogen homeostasis [6-8].

The association between MetS and the increased risk for atherosclerosis, cardiovascular disease (CVD) and type 2 diabetes are well known $[2,3,9]$. Furthermore, the triad of dyslipidemia i.e. hypertriglyceridemia, increased Low-Density Lipoprotein-Cholesterol (LDL-C) and decreased High-Density Lipoprotein-Cholesterol (HDLC) observed in MetS, introduces an underestimation of the risk for cardiovascular disease (CVD) using only LDL-C as a surrogate marker [10].

Recently, non-High-Density Lipoprotein-Cholesterol (non-HDL-C) and/or Apolipoprotein B (ApoB) were added as new biomarkers for CVD risk assessment according to guidelines from European Society of Cardiology (ESC) and European Society of Atherosclerosis (EAS) [11]. The latest biomarker to be introduced is remnant-Cholesterol (remnant-C), which includes the cholesterol in Triglyceride (TG)-Rich Lipoproteins (TGRLs)-Very Low-Density lipoprotein (VLDL), Intermediate-Density Lipoprotein (IDL) and Chylomicron remnant $[12,13]$.

In addition, ratios between lipoproteins and HDL-C have also been suggested to better mirror the balance between pro- and antiatherogenic components in serum, and to add value in CVD risk estimation, particularly for patients with MetS [14]. In fact, the HDL-C ratios of triglycerides (TG/HDL-C) have been suggested as a biomarker of MetS in both females and males [15].

Furthermore, trimethylamine-N-Oxide (TMAO), a metabolite originating from microbial metabolism in the gut but also from the diet, has been shown to be associated with cardiovascular events and also to have direct pro-atherosclerotic properties [16]. Moreover, plasma TMAO levels has been found to be an independent risk factor for CVD-associated mortality in MetS patients [17]. The gut derived metabolites betaine and choline have also been suggested as biomarkers for CVD $[16,18]$.

Several studies have shown fluctuations in lipids and other cardiometabolic markers, during the menstrual cycle, reflecting the influence of both sex and gender [19-23]. Interestingly, in a previous study on fertile women, acute myocardial infarction was reported to be more common during the follicular phase [24]. However, most studies of gut microbiota and their role in CVD have been performed in males and there is to our knowledge no published studies on menstrual cycle variability of these biomarkers in humans [16]. Finally, in order to evaluate the utility of novel biomarkers, studies are ideally performed in healthy volunteers prior to investigations in patient populations [25].

Thus, the primary aim of this pilot-study was to evaluate variations in levels and ratios of non-HDL-C, ApoB, remnant- $\mathrm{C}$ and in gut microbiota TMAO, betaine and choline, in healthy volunteers during two menstrual phases. The secondary aim was to investigate possible correlations between these markers and estrogen and progesterone levels, and the correlation between the biomarkers for lipids and microbial metabolites.

\section{Methods \\ Study population}

Serum samples were retrieved from a recently performed prospective cohort study of 17 healthy women with regular menses [26, 27]. The original study was aimed at investigating menstrual cycle variability in serum and urinary concentrations in pharmacological biomarkers of CYP3A-activity and of biomarkers included in the athlete biological passport (ABP). In addition, the lipid profile in the different menstrual phases in relation to serum sex hormones levels was also studied. The power calculations were based on the number of subjects needed to show an association between menstrual cycle phases and the urinary epitestosterone used in ABP. Finally, the investigation was performed at the outpatient clinic at the Department of Clinical Pharmacology, Karolinska Hospital, Stockholm, Sweden.

In summary, females aged 18-45 years with regular menses as defined by cycle length $24-38$ days, with vaginal bleeding $4-8$ days and cycle variation $\leq 7-9$ days were included [28]. Nineteen subjects were included in October and November 2018 after written informed consent and control of eligibility criteria. Two subjects later withdrew their consent due to lack of time to participate. Unfortunately, we were not able to recruit the 30 subjects needed for a $99 \%$ power as described above.

Subjects using oral contraceptives, who were breastfeeding or who were pregnant during the last three months were excluded. A physical examination was performed by a physician, including measurement of body mass index (BMI) and blood pressure.

No drugs containing hormones or drugs otherwise affecting the endocrine system were allowed. Over the counter vitamin supplements, and occasional NonSteroidal Anti-inflammatory Drugs (NSAID) and paracetamol during the menstrual period were allowed. Other regular medication was allowed as judged by the investigator.

Blood samples in total $14 \mathrm{ml}$ (one $4 \mathrm{ml}$ EDTA and two $5 \mathrm{ml}$ serum tube) were collected once a week during two menstrual cycles. Using this approach, sampling would capture the menstrual phase the subject was in at the time of investigation, based on an algorithm. The sampling procedure was done between 7 and $10 \mathrm{AM}$, and subjects were in a non-fasting state in order to reflect the atherogenic lipid profile in subjects over a 24 -h period. 
Depending on cycle length, every subject had between six to nine blood tests. Subjects monitored their bleeding pattern throughout the two cycles with an app or on paper.

\section{Laboratory analyses}

Routine safety parameters-hemoglobin, blood cells, c-reactive protein (CRP), albumin and creatinine were analyzed by the Laboratory of Clinical Chemistry, Karolinska University Hospital, Solna Sweden with ISO 15189:2012 accredited methods. The Karolinska Laboratory Medical Study Centre, a facility performing analysis of research samples according to Good Clinical Practice (GCP) standards, conducted analysis of the remaining blood samples. Serum was separated by centrifugation and stored at $-80^{\circ} \mathrm{C}$ until analysis.

Progesterone, and estrogen were determined by radioimmunoassay while luteinizing hormone (LH) and follicle stimulating hormone (FSH) were determined using electrochemiluminescence immunoassay. TG, total cholesterol (TC) and HDL-C were analyzed by enzymatic assay followed by photometry. Apolipoprotein A1 (ApoA1) and $A p o B$ were analyzed by immunochemistry followed by turbidimetry. LDL-C was calculated according to Friedewald, Levy and Fredrickson [29] while nonHDL-C and remnant- $C$ were calculated as described by Carr et al. [10].

\section{Analysis of TMAO, betaine and choline}

Analysis of TMAO, choline and betaine in serum samples were performed with LC-MS/MS at Swedish Metabolomic Centre in Umeå, Sweden using a protocol previously described [30].

\section{Definition of menstrual cycle phases}

The participants phase in the menstrual cycle at the time of each blood sampling was established using the algorithm:

Follicular phase defined as estrogen $<81 \mathrm{pg} / \mathrm{mL}$, progesterone $<1.6 \mathrm{ng} / \mathrm{mL}$ and FSH and $\mathrm{LH}$.

Ovulatory phase defined as estrogen $\geq 81 \mathrm{pg} / \mathrm{mL}$, progesterone $<1.6 \mathrm{ng} / \mathrm{mL}$ and LH higher than FSH. Finally, luteal phase defined as progesterone $>5.3 \mathrm{ng} / \mathrm{mL}$ [31].

\section{Statistical analysis}

All analyses were done in Stata 15 (Stata Corp 2017. Stata Statistical Software: Release 15. College Station, TX: StataCorp LLC.) The comparisons between different phases of the menstrual cycle were done with linear regression, sub-setting the data to include two phases at the time. The dependencies between different variables were also investigated with linear regression. Finally, the cluster robust covariance estimator was used in all regression analysis to account for the inter-person correlation caused by repeated measurements. In this manner we adjust for some subjects having two complete menstrual cycles and some only one.

\section{Results}

Of the 17 participants, 13 had two complete menstrual cycles, including the three phases follicular, ovulatory and luteal, according to the hormone levels measured in the samples. Thus, four participants contributed with only one complete menstrual cycle, including the three phases. Although data from the follicular and luteal phases were available for two cycles in these four subjects, the sampling had failed to capture the short ovulatory phase, as it lasts only $24 \mathrm{~h}$.

None of the subjects were smokers, and none used other forms of tobacco. Six subjects did not use alcohol, and eleven subjects had low alcohol intake once or twice per week. Fourteen subjects exercised between two to seven days per week (mean 3.3 days per week), while three exercised once a week.

Mean baseline values for the subjects at inclusion were age 33.2 years, BMI $22.0 \mathrm{~kg} / \mathrm{m}^{2}$, blood pressure $114 / 76 \mathrm{~mm} \mathrm{Hg}$ and pulse 76 beats per minute. During the study, mean bleeding days were 7 and the mean cycle length was 24.5 days. There were no significant changes in safety laboratory parameters during the study.

In total, blood samples from 30 complete menstrual cycles, including follicular, luteal and ovarian phases, could be retrieved from 17 women. Thus, 90 blood samples were analyzed for sex-hormones, lipids, TMAO, betaine and choline.

\section{Sex hormones}

To confirm known variations in sex hormones during the menstrual cycle, levels of estrogen and progesterone, as well as FSH and LH, were controlled. For estrogen there were significant differences between all phases (Fig. 1a), whereas progesterone was significantly higher in the luteal phase (Fig. 1b).

\section{Lipids and lipoproteins}

Levels of HDL-C and TG were higher during the luteal phase when estrogen levels are higher (Fig. 2). Levels of the new biomarkers ApoB, ApoB/HDL and non-HDL-C/ HDL ratio were highest during the follicular phase, while remnant- $\mathrm{C}$ levels were highest during the luteal phase (Fig. 2).

Low levels of estrogen were associated with higher levels of non-HDL-C and ApoB (Fig. 3). Likewise, low levels of estrogen were associated with higher levels of the lipid ratios non-HDL-C/HDL, and ApoB/HDL (Fig. 3). 

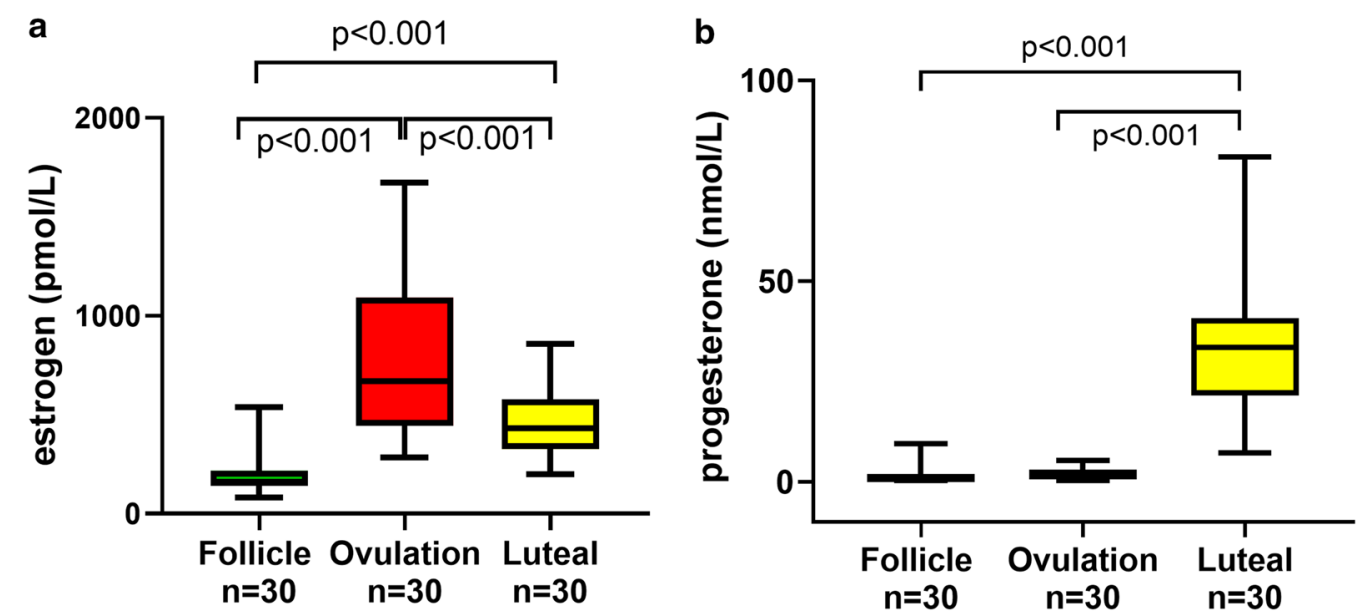

Fig. 1 a, b Variations in estrogen and progesterone throughout the different phases in 30 menstrual cycles from 17 fertile women

There was no correlation between estrogen and remnant-C or the remnant -C/HDL ratio (Fig. 3). No correlation was found between any of the lipids, lipoproteins or ratios and progesterone levels.

To summarize, higher levels of pro-atherogenic lipoproteins and their HDL-ratios was observed during the follicular phase, while remnant- $C$ increased during the luteal phase. Significant correlation between the ratios for dyslipidemia and estrogen was shown, with higher levels of pro-atherogenic biomarkers associated with low estrogen levels.

\section{TMAO, betaine and choline}

There were no significant differences in TMAO and Choline levels during follicular, ovulatory and luteal phases. In contrast, betaine levels were higher in the ovulatory phase compared to the luteal phase $(p<0.05)$ (Table 1 , Fig. 4). None of the microbiota metabolites showed any correlation with estrogen or with progesterone levels.

\section{Correlation between TMAO, choline, betaine and non-HDL-C, remnant- $C$ and $A p o B$ levels}

There were no significant correlations between TMAO or choline and lipids or lipid ratios (Fig. 5). There was a weak association between betaine and non-HDL-C; $p<0.05$ (Fig. 5). To conclude, no strong association between the gut microbiota metabolites and the new lipid biomarkers were observed.

\section{Discussion}

In this study, we show that low estrogen levels, as present during the follicular phase of the menstrual cycle, was associated with higher levels of proatherogenic non-HDL-C, and ApoB and their HDL-ratios.
Remnant- $C$ was higher during the luteal phase and showed no correlation with estrogen levels. The proatherogenic microbial metabolite TMAO did not show any association with levels of sex hormones or lipid biomarkers.

The results of our study on menstrual cycle variability in lipids and their ratios are in line with previously performed studies with higher levels of proatherogenic lipids during the follicular phase compared to luteal phase [20-22]. The HDL-C levels were higher during the luteal phase when estrogen is high [32]. The decrease in $A p o B$ and $A p o B / H D L$ ratio in the ovulatory and luteal phase of our study, has not been observed previously [33, 34]. However, this is in agreement with previous findings of decreased atherogenicity during the luteal phase [20].

The significant increase in remnant- $\mathrm{C}$ and its surrogate marker TG [13] during the luteal phase, could be due to the non-fasting sampling in the present study. However, as sampling in the other phases were performed in the same manner, this is not likely to be the cause. Another possible explanation is the increase in prevalence of high TG and MetS in the general population, compared to lipid studies performed 20 to 30 years ago. Mendelian randomization studies suggest that TG-rich lipoproteins like remnant- $C$ are causally associated with an increased risk of CVD and to all-cause mortality, particularly in women $[19,35,36]$. The implications of our study could be that sampling for evaluating risk in patients with MetS and/or high TG, may be performed not only during the follicular phase but also during the luteal phase. If remnant-C/HDL ratio is evaluated, sampling during the follicular phase could be sufficient. However, there are no established reference 

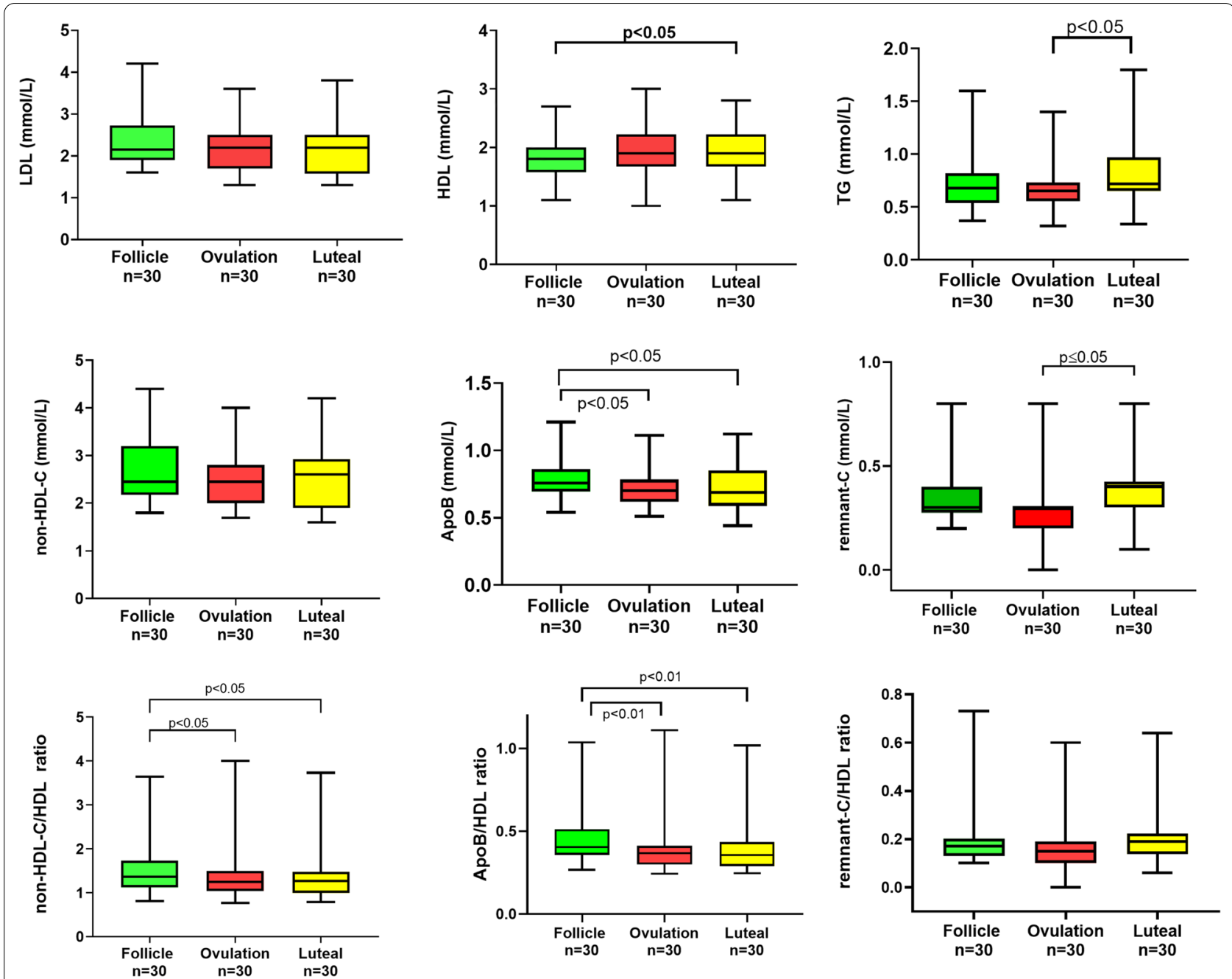

Fig. 2 Variations in Low-Density Lipoprotein-Cholesterol (LDL-C), High-Density Lipoprotein-Cholesterol (HDL-C), Triglycerides (TG),

non-High-Density Lipoprotein-Cholesterol (non-HDL-C), remnant-Cholesterol (remnant-C) and Apolipoprotein B (ApoB) levels and their HDL-ratios throughout follicular, ovulatory and luteal phases of 30 menstrual cycles from 17 fertile women

values for the ratios non-HDL-C/HDL, ApoB/HDL and remnant-C/HDL in adults, as opposed to age- and/or sex-specific values for levels of non-HDL-C, remnant-C and ApoB respectively [37].

It has been suggested that a combination of biomarkers will improve the CVD assessment and the newly added biomarkers give different contributions to such an evaluation. Non-HDL-C includes Lp(a), a more powerful predictor of CVD incidence in women compared to men [8]. Non-HDL-C also confer information on total cholesterol content, measured as mass. ApoB is a measure of the number of atherogenic particles in the circulation [10] while remnant- $\mathrm{C}$ provides information on the cholesterol content in the postprandial triglyceride-rich lipoproteins (TGRLs) [13].
The results from our study do not support a strong association between estrogen and the levels of the microbial metabolites TMAO, betaine or choline in blood. In a previous study of 25 males and 7 postmenopausal women, a strong association was found between levels of estrogens and their metabolites in urine and the diversity of microbiota in feces [38]. However, no association for premenopausal women was found in the study, which is concurring with our results. This could also be related to the sampling of the fertile women, being performed irrespective of menstrual cycle phase, resulting in highly variable estrogen levels. Interestingly, in a review studies of fecal microbiota transplant studies to patients with MetS and obesity, none of the 76 included patients were female [39]. 

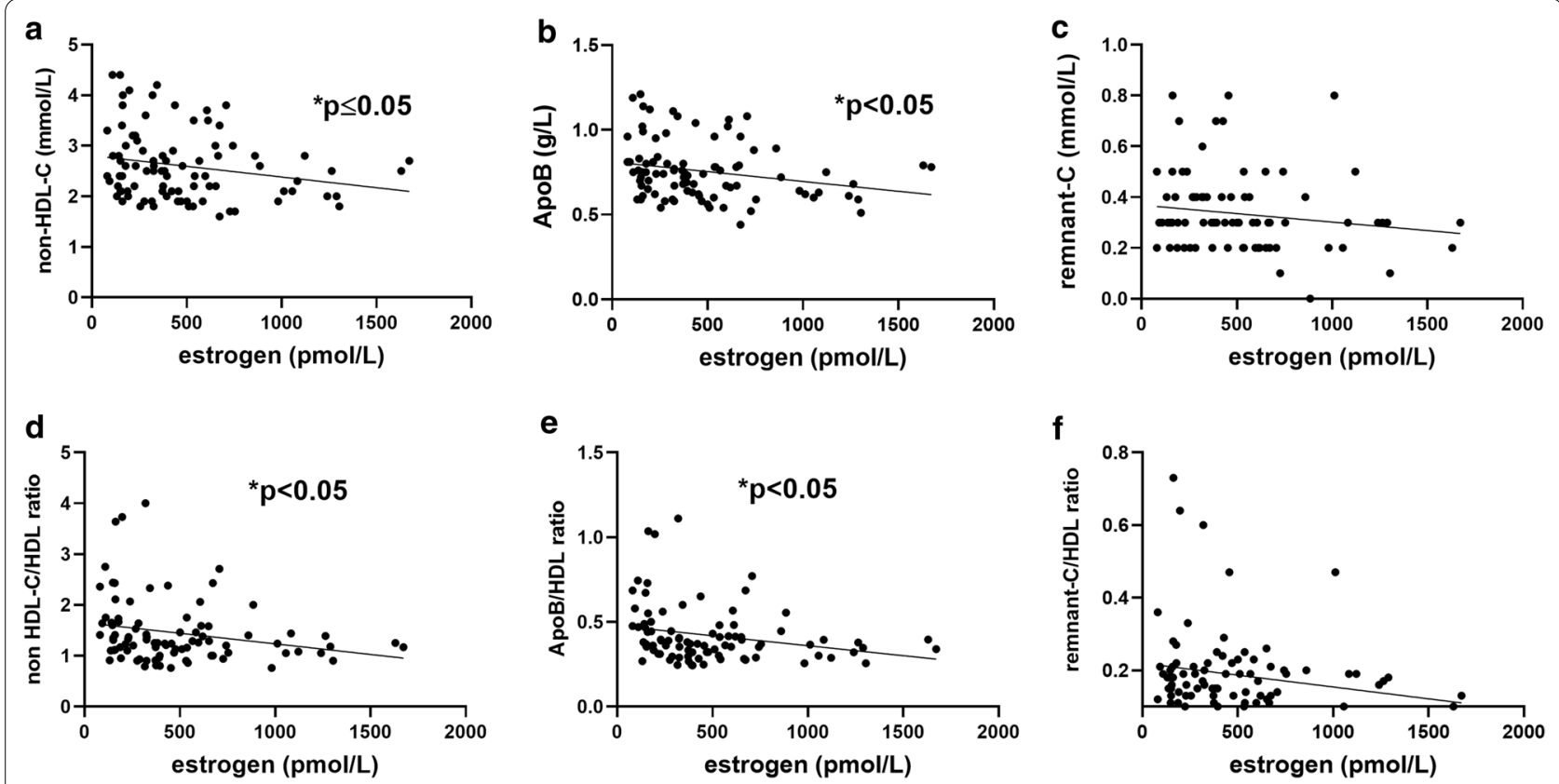

Fig. 3 Correlation analysis between estrogen levels and biomarkers for dysplipidemia: a non-High-Density Lipoprotein-Cholesterol (non-HDL-C), b Apolipoprotein B (ApoB), c remnant-Cholesterol (remnant-C) and $\mathbf{d}-\mathbf{f}$ their HDL-ratios throughout follicular, ovulatory and luteal phases of 30 menstrual cycles from 17 fertile women

Table 1 Plasma levels of novel biomarkers for dysplipidemia and dysbiosis during the follicular, ovulatory and luteal phase of 30 menstrual cycles in 17 regularly menstruating women

\begin{tabular}{llll}
\hline Parameter & $\begin{array}{l}\text { Follicular phase } \\
\text { Mean } \pm \text { SD } \\
\text { Median }\end{array}$ & $\begin{array}{l}\text { Ovulatory phase } \\
\text { Mean } \pm \text { SD } \\
\text { Median }\end{array}$ & $\begin{array}{l}\text { Luteal phase } \\
\text { Mean } \pm \text { SD } \\
\text { Median }\end{array}$ \\
\hline TMAO $(\mathrm{ng} / \mathrm{\mu L})$ & $0.35( \pm 0.33)$ & $0.31( \pm 0.24)$ & $0.25( \pm 0.14)$ \\
& 0.25 & 0.21 & 0.22 \\
Betaine $(\mathrm{ng} / \mathrm{\mu L})$ & $3.99( \pm 10.7)$ & $4.20( \pm 1.01)$ & $3.81( \pm 1.12)$ \\
& 4.11 & 4.13 & 3.67 \\
Choline $(\mathrm{ng} / \mathrm{HL})$ & $4.98( \pm 0.85)$ & $5.15( \pm 0.87)$ & $4.93( \pm 1.00)$ \\
& 4.79 & 4.98 & 4.96 \\
Non-HDL-C & $2.7( \pm 0.72)$ & $2.51( \pm 0.64)$ & $2.60( \pm 0.71)$ \\
(mmol/L) & 2.45 & 2.45 & 2.60 \\
Remnant-C & $0.33( \pm 0.12)$ & $0.30( \pm 0.15)$ & $0.38( \pm 0.17)$ \\
(mmol/L) & 0.30 & 0.30 & 0.40 \\
ApoB $(\mathrm{g} / \mathrm{L})$ & $0.80( \pm 0.17)$ & $0.73( \pm 0.16)$ & $0.74( \pm 0.18)$ \\
& 0.75 & 0.70 & 0.68 \\
Non-HDL-C/HDL & $1.56( \pm 0.63)$ & $1.40( \pm 0.67)$ & $1.40( \pm 0.59)$ \\
& 1.36 & 1.24 & 1.27 \\
ApoB/HDL & $0.46( \pm 0.17)$ & $0.41( \pm 0.18)$ & $0.40( \pm 0.15)$ \\
& 0.40 & 0.38 & 0.36 \\
Remnant-C/HDL & $0.19( \pm 0.12)$ & $0.17( \pm 0.12)$ & $0.21( \pm 0.12)$ \\
& 0.17 & 0.15 & 0.19 \\
\hline
\end{tabular}

TMAO, trimethylamine N-oxide; non-HDL-C, non-High-Density LipoproteinCholesterol; remnant-C, remnant-Cholesterol; ApoB, Apolipoprotein B
Notably, no correlation between TMAO and the new lipid biomarkers were observed in this study. Still, TMAO appears to play an important role in cholesterol metabolism, causing inhibition of reverse cholesterol transport and an increase in foam cell activity by activating macrophages in the immune system [16]. In addition, TMAO may repress bile acid synthesis, thus effecting the major pathway for cholesterol elimination [16]. TMAO homeostasis is complex and is not only affected by gut microbiota but also by e.g. diet, age, gender, BMI and kidney function [40].

The link between the gut microbiota and CVD has become stronger in recent years [40]. However, TMAO is still not validated for clinical use in CVD risk assessment and a study of healthy middle-aged adults, did not find any association between TMAO and the risk of advancing atherosclerosis [41].

The strength of our study is that the exact menstrual phases were verified by measuring FSH, LH, estrogen and progesterone on a weekly basis. Also, lipids were measured at the same time point as sex hormones. Furthermore, a novelty in our study was that the sampling was performed in a non-fasting state in accordance with recent consensus [12] and in contrast with most previously performed studies [22]. In this manner, triglyceride-rich lipoproteins were also included in the lipid profile. Not controlling for dietary intake, also increases the probability of having a dietary status more 

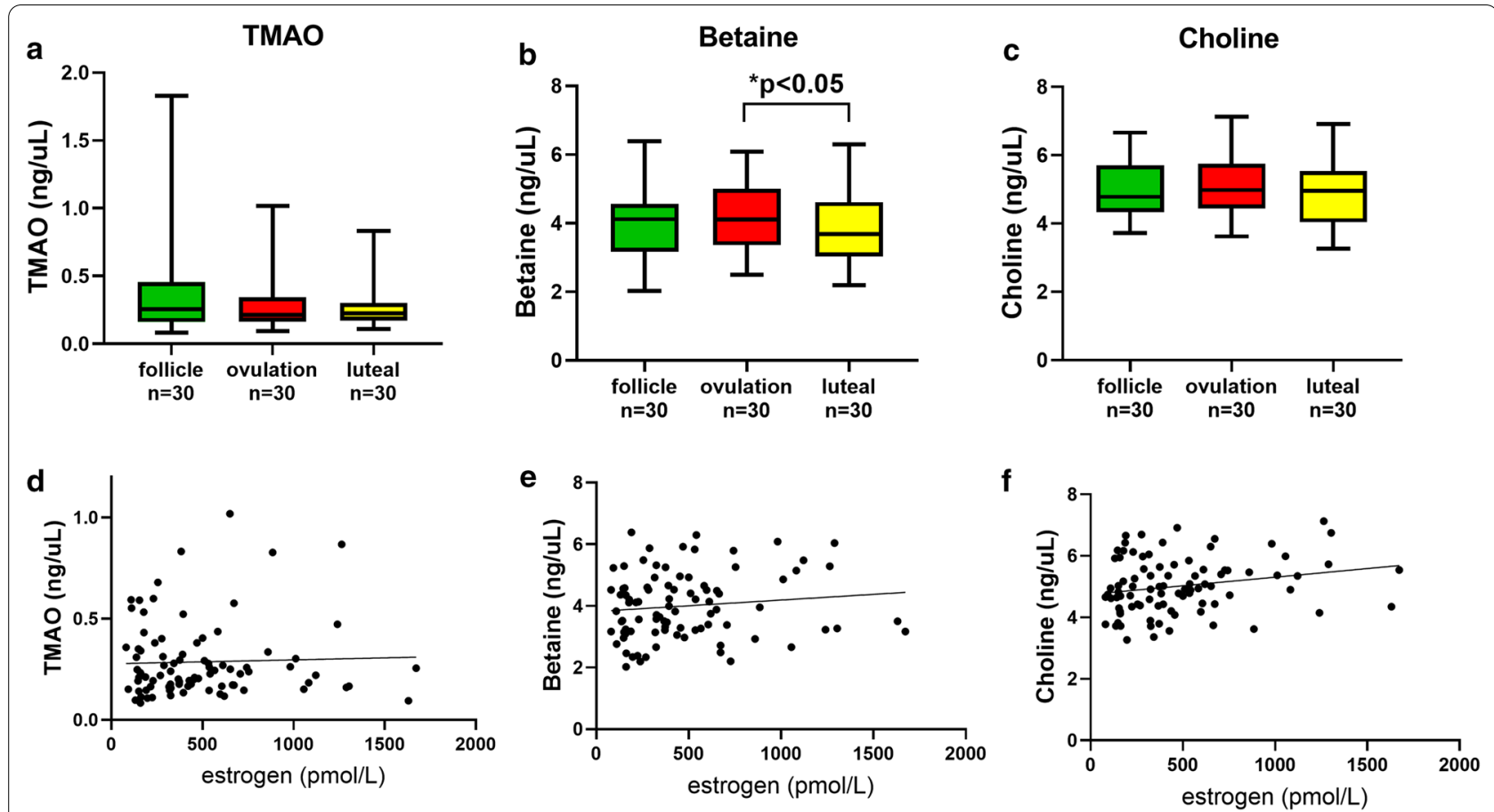

Fig. 4 Variations in levels of gut microbiota metabolites a trimethylamine-N-Oxide (TMAO), $\mathbf{b}$ betaine and $\mathbf{c}$ choline in plasma throughout the different phases in 30 menstrual cycles from 17 fertile women and $\mathbf{d}-\mathbf{f}$ shows correlation analysis between the metabolites and estrogen $(n=90)$

representative of ordinary life. Data on relevant lifestyle factors like physical activity, smoking/snuff and alcohol intake that may affect lipid homeostasis, were also registered. This study is also the first describing the levels of gut microbiota metabolites in the circulation in healthy, fertile women in the different menstrual cycle phases.

However, a major limitation in this study is the small sample size. Thus, the findings should be confirmed in a future study with a larger sample size. In addition, standardization of diet could be considered in order to evaluate whether dietary intake of choline and betaine affect our findings between these markers and lipids [18]. Finally, the use of calculated LDL and remnant-C may introduce error sources. There are currently no commercially available direct assays method for remnant- $\mathrm{C}$, but there is for LDL, although not commonly used in routine clinical practice [12].

The next step would be to investigate the biomarkers for dyslipidemia and dysbiosis in a patient cohort with MetS, but without polycystic ovary syndrome, during the menstrual cycle. Further elucidating the connections between hormones, microbiota and dyslipidemia may contribute to better treatment options for women, both pharmacological and non-pharmacological.

In recent years it has been suggested that B-vitamins and omega-3-polyunsaturated fatty acids may affect dyslipidemia and dysbiosis [42, 43]. In future studies, performed on women with metabolic syndrome or other risk factor for CVD, it would be interesting to study the interplay between these nutrients and the biomarkers studied here.

Vitamin $\mathrm{D}$ has been shown to affect endometrial function and low levels of vitamin D is associated with impaired fertility and Polycystic Ovary Syndrome (PCOS) [44, 45]. In addition, Vitamin D deficiency has been shown to be associated with high levels of TMAO and obesity [46]. In future studies it would be interesting to study the relationship between Vitamin D and TMAO in women with PCOS and if it affects the risk of CVD. The results from the present study, including healthy, fertile women without PCOS, could constitute a valuable reference cohort for such studies.

\section{Conclusion}

For the first time, we have analyzed the levels of different microbial metabolites in blood during the menstrual cycle. Furthermore, our data supports that the new biomarkers recommended for estimation of CVD-risk and as treatment targets in dyslipidemia, vary during the menstrual cycle.

The increased prevalence in obesity, metabolic syndrome and CVD in fertile, menstruating women, indicate that in years to come, there will be a high need for new, additional biomarkers in combination to optimize CVD 


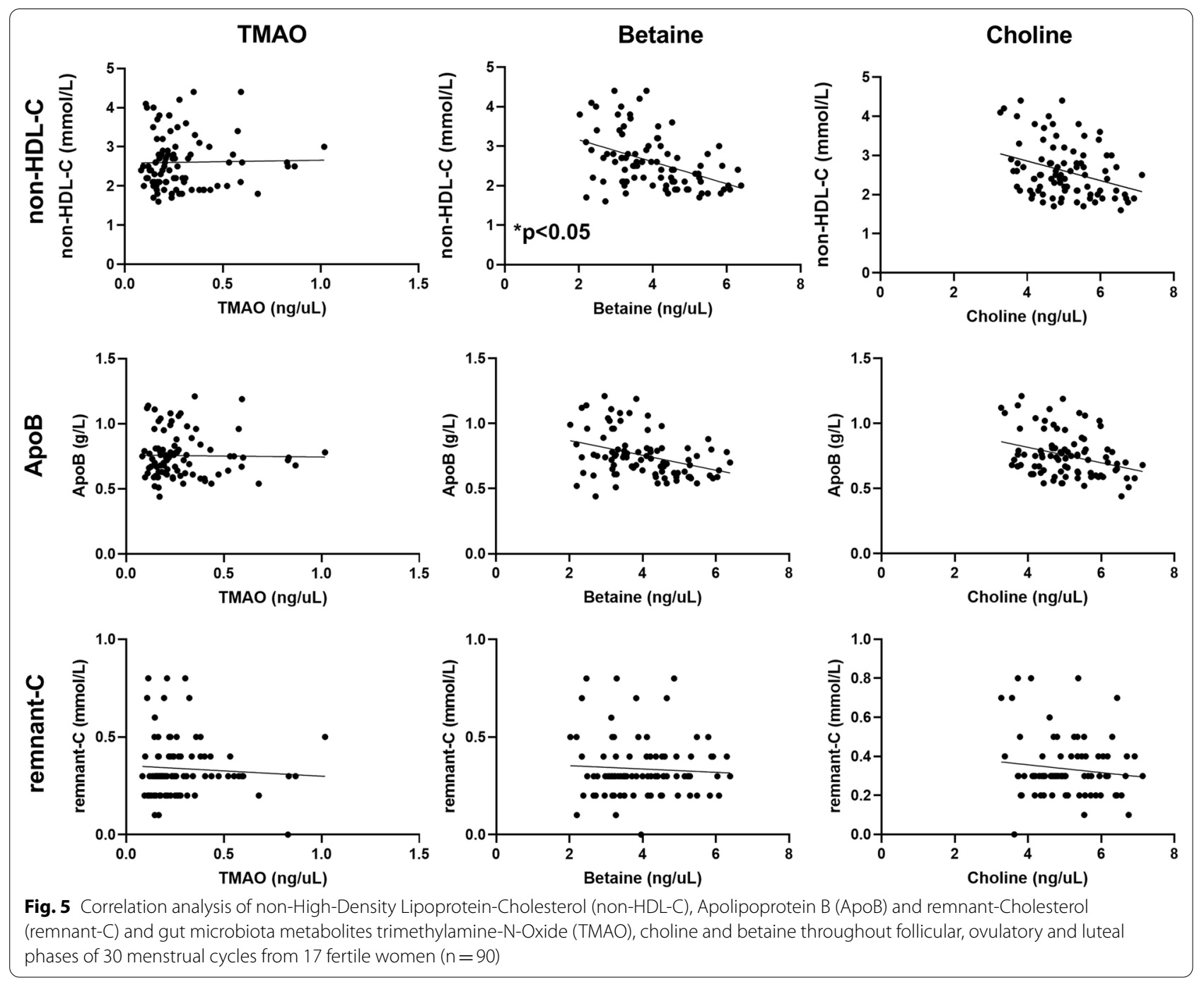

assessments and treatment in this population. Further studies of menstrual cycle variability in fertile women with metabolic syndrome/type 2 diabetes and previous CVD, is warranted.

\footnotetext{
Abbreviations

CVD: Cardiovascular disease; FSH: Follicle stimulating hormone; LH: Luteinizing hormone; LDL-C: Low-Density Lipoprotein-Cholesterol; HDL-C: High-Density Lipoprotein-Cholesterol; Non-HDL-C: Non-HDL cholesterol; TC: Total Cholesterol; ApoB: Apolipoprotein B; ApoA1: Apolipoprotein A1; Remnant-C: Remnant-Cholesterol; TGRLs: Triglyceride-Rich Lipoproteins; VLDL: Very Low-Density Lipoprotein; IDL: Intermediate-Density Lipoprotein; TMAO Trimethylamine-N-Oxide; MetS: Metabolic syndrome; AMI: Acute myocardial infarction; ESC: European Society of Cardiology; EAS: European Society of Atherosclerosis; ABP: Athletes biological passport; CRP: C-reactive protein; BMI: Body mass index; NSAID: Non-steroidal anti-inflammatory drug; GCP: Good Clinical Practice; PCOS: Polycystic Ovary Syndrome.
}

\section{Acknowledgements}

The authors would like to express their sincere gratitude to all healthy volunteers who participated in the study. The authors are also grateful to study nurse Susanne Broström for skillful work with the study and Annika Johansson at The Swedish Metabolomic Centre, Swedish Agricultural University, Umeå, Sweden, for technical assistance with the analyses of gut microbiota.

\section{Authors' contributions}

$L B B, H B, P B$ and $L E$ were responsible for study design, funding applications, applications to Swedish Ethical Review Authority, Biobank Sweden and Swedish Data Protection Authority. Recruiting and screening was performed by LBB as study physician and LE. HB compiled data from laboratory reports and CRFs. HB performed statistical analysis together with statistician AW and LBB. HB wrote original draft of the manuscript. All read and approved the final manuscript.

\section{Authors' information}

Helena Bergström is MD and specialist in Clinical Pharmacology and Family Medicine and PhD-student at Karolinska Institutet.

Lena Ekström is PhD and Associate Professor in Clinical Pharmacology at Karolinska Institutet.

\section{Anna Warnqvist is Biostatistician at Karolinska Institutet.}


Peter Bergman is MD and specialist in Clinical Microbiology and Associate Professor at Karolinska Institutet.

Linda Björkhem-Bergman is MD, and a specialist in Clinical Pharmacology and Palliative Medicine and Associate Professor at Karolinska Institutet.

\section{Funding}

Open access funding provided by Karolinska Institute. The present study was funded by the Swedish Research Council for Sport Sciences (CIF) (P20180065), Stockholm County Council (ALF 20160036 and 20180320), the Swedish Cancer Society (CAN 2017/233 and CAN 2018/316).

\section{Availability of data and materials}

As sample size is small, there is a risk of the individual privacy being compromised. De-identified data are therefore available from the corresponding author upon reasonable request.

\section{Declarations}

\section{Ethics approval and consent to participate}

The study was approved by the Swedish Ethical Review Authority (Dnr 2018/481-31/2 and Dnr 2020-00598) and was performed in accordance with the Declaration of Helsinki. Written informed consent was obtained from all patients prior to inclusion in the study.

\section{Consent for publication}

Not applicable.

\section{Competing interests}

The authors have no conflict of interest to disclose.

\section{Author details}

${ }^{1}$ Division of Clinical Geriatrics, Department of Neurobiology, Care Sciences and Society (NVS), Karolinska Institute, Blickagången 16, Neo floor 7, 141 83 Huddinge, Sweden. ${ }^{2}$ Division of Clinical Pharmacology, Department of Laboratory Medicine, Karolinska Institutet, and Karolinska University Laboratory, Karolinska University Hospital, 14183 Huddinge, Sweden. ${ }^{3}$ Division of Biostatistics, Department of Environmental Medicine, Karolinska Institutet, Nobels väg 13, 17177 Stockholm, Sweden. ${ }^{4}$ Division of Clinical Microbiology, Department of Laboratory Medicine, Karolinska Institutet, ANA Futura, Alfred Nobels Allé 8, 14152 Huddinge, Sweden. ${ }^{5}$ Department of Infectious Diseases, Immunodeficiency Unit, Karolinska University Hospital, 14183 Huddinge, Sweden. ${ }^{6}$ Stockholms Sjukhem, Palliative Medicine, Mariebergsgatan 22, 112 19 Stockholm, Sweden.

\section{Received: 16 December 2020 Accepted: 14 April 2021} Published online: 20 April 2021

\section{References}

1. Benjamin EJ, Muntner P, Alonso A, Bittencourt MS, Callaway CW, Carson AP, et al. Heart disease and stroke statistics-2019 update: a report from the American Heart Association. Circulation. 2019;139(10):e56-528.

2. Pucci G, Alcidi R, Tap L, Battista F, Mattace-Raso F, Schillaci G. Sex- and gender-related prevalence, cardiovascular risk and therapeutic approach in metabolic syndrome: a review of the literature. Pharmacol Res. 2017; 120:34-42.

3. Gerdts E, Regitz-Zagrosek V. Sex differences in cardiometabolic disorders. Nat Med. 2019;25(11):1657-66.

4. Towfighi A, Zheng L, Ovbiagele B. Sex-specific trends in midlife coronary heart disease risk and prevalence. Arch Intern Med. 2009;169(19):1762-6.

5. Santos-Marcos JA, Haro C, Vega-Rojas A, Alcala-Diaz JF, Molina-Abril H, Leon-Acuna A, et al. Sex differences in the gut microbiota as potential determinants of gender predisposition to disease. Mol Nutr Food Res. 2019;63(7):e1800870.

6. Schoeler M, Caesar R. Dietary lipids, gut microbiota and lipid metabolism. Rev Endocr Metab Disord. 2019;20(4):461-72.

7. Link JC, Reue K. Genetic basis for sex differences in obesity and lipid metabolism. Annu Rev Nutr. 2017;37:225-45.
8. Baker JM, Al-Nakkash L, Herbst-Kralovetz MM. Estrogen-gut microbiome axis: physiological and clinical implications. Maturitas. 2017;103:45-53.

9. Aboonabi A, Meyer RR, Singh I. The association between metabolic syndrome components and the development of atherosclerosis. J Hum Hypertens. 2019;33(12):844-55.

10. Carr SS, Hooper AJ, Sullivan DR, Burnett JR. Non-HDL-cholesterol and apolipoprotein B compared with LDL-cholesterol in atherosclerotic cardiovascular disease risk assessment. Pathology. 2019;51(2):148-54.

11. Mach F, Baigent C, Catapano AL, Koskinas KC, Casula M, Badimon L, et al. 2019 ESC/EAS Guidelines for the management of dyslipidaemias: lipid modification to reduce cardiovascular risk. Eur Heart J. 2020;41(1):111-88.

12. Nordestgaard BG, Langlois MR, Langsted A, Chapman MJ, Aakre KM, Baum $\mathrm{H}$, et al. Quantifying atherogenic lipoproteins for lipid-lowering strategies: consensus-based recommendations from EAS and EFLM. Atherosclerosis. 2020;294:46-61.

13. Sandesara PB, Virani SS, Fazio S, Shapiro MD. The Forgotten lipids: triglycerides, remnant cholesterol, and atherosclerotic cardiovascular disease risk. Endocr Rev. 2019;40(2):537-57.

14. Millán J, Pintó X, Muñoz A, Zúñiga M, Rubiés-Prat J, Pallardo LF, et al. Lipoprotein ratios: physiological significance and clinical usefulness in cardiovascular prevention. Vasc Health Risk Manag. 2009;5:757-65.

15. Gasevic D, Frohlich J, Mancini GJ, Lear SA. Clinical usefulness of lipid ratios to identify men and women with metabolic syndrome: a cross-sectional study. Lipids Health Dis. 2014;13:159.

16. Liu Y, Dai M. Trimethylamine N-oxide generated by the gut microbiota is associated with vascular inflammation: new insights into atherosclerosis. Mediators Inflamm. 2020;2020:4634172.

17. Tang WH, Wang Z, Li XS, Fan Y, Li DS, Wu Y, et al. Increased trimethylamine $\mathrm{N}$-oxide portends high mortality risk independent of glycemic control in patients with type 2 diabetes mellitus. Clin Chem. 2017;63(1):297-306.

18. Millard HR, Musani SK, Dibaba DT, Talegawkar SA, Taylor HA, Tucker KL, et al. Dietary choline and betaine; associations with subclinical markers of cardiovascular disease risk and incidence of CVD, coronary heart disease and stroke: the Jackson Heart Study. Eur J Nutr. 2018;57(1):51-60.

19. Palmisano BT, Zhu L, Eckel RH, Stafford JM. Sex differences in lipid and lipoprotein metabolism. Molecular metabolism. 2018;15:45-55.

20. Vashishta S, Gahlot S, Goyal R. Effect of menstrual cycle phases on plasma lipid and lipoprotein levels in regularly menstruating women. J Clin Diagn Res. 2017;11(5):Cc05-cc7.

21. Mumford SL, Schisterman EF, Siega-Riz AM, Browne RW, Gaskins AJ, Trevisan $M$, et al. A longitudinal study of serum lipoproteins in relation to endogenous reproductive hormones during the menstrual cycle: findings from the BioCycle study. J Clin Endocrinol Metab. 2010;95(9):E80-5.

22. Mumford SL, Dasharathy S, Pollack AZ, Schisterman EF. Variations in lipid levels according to menstrual cycle phase: clinical implications. Clinical lipidology. 2011;6(2):225-34.

23. Schisterman EF, Mumford SL, Sjaarda LA. Failure to consider the menstrua cycle phase may cause misinterpretation of clinical and research findings of cardiometabolic biomarkers in premenopausal women. Epidemiol Rev. 2014;36:71-82.

24. Mukamal KJ, Muller JE, Maclure M, Sherwood JB, Mittleman MA. Variation in the risk of onset of acute myocardial infarction during the menstrual cycle. Am J Cardiol. 2002;90(1):49-51.

25. Aronson JK, Ferner RE. Biomarkers-A general review. Curr Protoc Pharmacol. 2017;76:9.23.1-9.23.17.

26. Mullen J, Bækken L, Bergström H, Björkhem-Bergman L, Ericsson M, Ekström L. Fluctuations of hematological ABP biomarkers in relation to the menstrual cycle. Drug Test Anal. 2020;12:1229-40.

27. Schulze J, Suominen T, Bergström H, Ericsson M, Björkhem Bergman L, Ekström L. Urinary steroid profile in relation to the menstrual cycle. Drug Test Anal. 2020;13:550-7.

28. Munro MG, Critchley HOD, Fraser IS. The two FIGO systems for normal and abnormal uterine bleeding symptoms and classification of causes of abnormal uterine bleeding in the reproductive years: 2018 revisions. Int J Gynaecol Obstet. 2018;143(3):393-408.

29. Friedewald WT, Levy RI, Fredrickson DS. Estimation of the concentration of low-density lipoprotein cholesterol in plasma, without use of the preparative ultracentrifuge. Clin Chem. 1972;18(6):499-502.

30. Missailidis C, Hallqvist J, Qureshi AR, Barany P, Heimburger O, Lindholm $\mathrm{B}$, et al. Serum trimethylamine- $\mathrm{N}$-oxide is strongly related to renal 
function and predicts outcome in chronic kidney disease. PLOS ONE. 2016;1 (1):e0141738.

31. Jewelewicz R, Dyrenfurth I, Ferin M, Bogumil J, Vande Wiele RL. Gonadotropin, estrogen and progesterone response to long term gonadotropinreleasing hormone infusion at various stages of the menstrual cycle. J Clin Endocrinol Metab. 1977;45(4):662-7.

32. Barnett JB, Woods MN, Lamon-Fava S, Schaefer EJ, McNamara JR, Spiegelman D, et al. Plasma lipid and lipoprotein levels during the follicular and luteal phases of the menstrual cycle. J Clin Endocrinol Metab. 2004;89(2):776-82.

33. Muesing RA, Forman MR, Graubard BI, Beecher GR, Lanza E, McAdam PA, et al. Cyclic changes in lipoprotein and apolipoprotein levels during the menstrual cycle in healthy premenopausal women on a controlled diet. J Clin Endocrinol Metab. 1996;81(10):3599-603.

34. Magkos F, Patterson BW, Mittendorfer B. No effect of menstrual cycle phase on basal very-low-density lipoprotein triglyceride and apolipoprotein B-100 kinetics. Am J Physiol Endocrinol Metab. 2006;291(6):E1243-9.

35. Nordestgaard BG. Triglyceride-rich lipoproteins and atherosclerotic cardiovascular disease: new insights from epidemiology, genetics, and biology. Circ Res. 2016;118(4):547-63.

36. Varbo A, Benn M, Tybjaerg-Hansen A, Jorgensen AB, Frikke-Schmidt R, Nordestgaard BG. Remnant cholesterol as a causal risk factor for ischemic heart disease. J Am Coll Cardiol. 2013;61(4):427-36.

37. Ridefelt P, Hagstrom E, Svensson MK, Akerfeldt T, Larsson A. Age- and sex-specific reference values for non-HDL cholesterol and remnant cholesterol derived from the Nordic Reference Interval Project (NORIP). Scand J Clin Lab Invest. 2019;79(1-2):39-42.

38. Flores R, Shi J, Fuhrman B, Xu X, Veenstra TD, Gail MH, et al. Fecal microbial determinants of fecal and systemic estrogens and estrogen metabolites: a cross-sectional study. J Transl Med. 2012;10:253.

39. Zhang Z, Mocanu V, Cai C, Dang J, Slater L, Deehan EC, et al. Impact of fecal microbiota transplantation on obesity and metabolic syndrome-a systematic review. Nutrients. 2019;11(10):2291.
40. Yang S, Li X, Yang F, Zhao R, Pan X, Liang J, et al. Gut microbiota-dependent marker TMAO in promoting cardiovascular disease: inflammation mechanism, clinical prognostic, and potential as a therapeutic target. Front Pharmacol. 2019;10:1360.

41. Meyer KA, Benton TZ, Bennett BJ, Jacobs DR Jr, Lloyd-Jones DM, Gross $M D$, et al. Microbiota-dependent metabolite trimethylamine $\mathrm{N}$-oxide and coronary artery calcium in the Coronary Artery Risk Development in Young Adults Study (CARDIA). J Am Heart Assoc. 2016;5(10):e003970.

42. Rizzo G, Lagana AS. The link between homocysteine and omega-3 polyunsaturated fatty acid: critical appraisal and future directions. Biomolecules. 2020;10(2):219.

43. Rizzo G, Lagana AS, Rapisarda AM, La Ferrera GM, Buscema M, Rossetti P, et al. Vitamin B12 among vegetarians: status, assessment and supplementation. Nutrients. 2016;8(12):767.

44. Colonese F, Lagana AS, Colonese E, Sofo V, Salmeri FM, Granese R, et al. The pleiotropic effects of vitamin $D$ in gynaecological and obstetric diseases: an overview on a hot topic. Biomed Res Int. 2015;2015:986281.

45. Lagana AS, Vitale SG, Ban Frangez H, Vrtacnik-Bokal E, D'Anna R. Vitamin D in human reproduction: the more, the better? An evidence-based critical appraisal. Eur Rev Med Pharmacol Sci. 2017;21(18):4243-51.

46. Barrea L, Muscogiuri G, Annunziata G, Laudisio D, de Alteriis G, Tenore $\mathrm{GC}$, et al. A new light on vitamin D in obesity: a novel association with trimethylamine-N-oxide (TMAO). Nutrients. 2019;1 1(6):1310.

\section{Publisher's Note}

Springer Nature remains neutral with regard to jurisdictional claims in published maps and institutional affiliations.
Ready to submit your research? Choose BMC and benefit from:

- fast, convenient online submission

- thorough peer review by experienced researchers in your field

- rapid publication on acceptance

- support for research data, including large and complex data types

- gold Open Access which fosters wider collaboration and increased citations

- maximum visibility for your research: over 100M website views per year

At BMC, research is always in progress.

Learn more biomedcentral.com/submissions 